\title{
ON 2-STEP SOLVABLE GROUPS OF FINITE MORLEY RANK
}

\author{
KATHRYN ENOCHS AND ALI NESIN
}

(Communicated by Andreas R. Blass)

\begin{abstract}
We prove the following results: Theorem 1. Let $G$ be a connected, centerless, solvable group of class 2 and of finite Morley rank. Then we can interpret in $G$ finitely many connected, solvable of class 2 and centerless algebraic groups $\widetilde{G}_{1}, \ldots, \widetilde{G}_{n}$ over algebraically closed fields $K_{i}$ in such a way that $G$ interpretably imbeds in $\widetilde{G}=\widetilde{G}_{1} \oplus \cdots \oplus \widetilde{G}_{n}$. Furthermore, $G^{\prime}=(\widetilde{G})^{\prime}$. Let $F(G)$ denote the Fitting subgroup of $G$. Theorem 2. Let $G, \widetilde{G}, \widetilde{G}_{i}$ be as in Theorem 1. Then (i) $F(G)=F(\widetilde{G}) \cap G$. (ii) $F(G)$ has a complement $V$ in $G: G=F \rtimes V$. (iii) Elements of $F(G)$ are unipotent elements of $G$ in $\widetilde{G}$. (iv) If the characteristic of each base field $K_{i}$ of $\widetilde{G}_{i}$ is different from 0 , then $V$ is definable and its elements are semi-simple in $\widetilde{G}$.
\end{abstract}

\section{INTRODUCTION}

This is a continuation of [Ne]; two of the questions asked in that article will be answered.

Theorem 1. Let $G$ be a connected, centerless, solvable group of class 2 and of finite Morley rank. Then we can interpret in $G$ finitely many connected, solvable of class 2 and centerless algebraic groups $\widetilde{G}_{1}, \ldots, \widetilde{G}_{n}$ over algebraically closed fields $K_{i}$ in such a way that $G$ definably imbeds in $\widetilde{G}=\widetilde{G}_{1} \oplus \cdots \oplus \widetilde{G}_{n}$. Furthermore $G^{\prime}=(\widetilde{G})^{\prime}$.

The groups $\widetilde{G}_{i}$ were defined in [Ne] and in fact most of the Theorem 1 was proved in $[\mathrm{Ne}]$. We only need to prove the algebraicity of each $\widetilde{G}_{i}$.

Let $\widetilde{G}, \widetilde{G}_{i}$ be as in the conclusion of Theorem 1 . We say that an element $g \in \widetilde{G}$ is unipotent (resp. semi-simple) if $g=g_{1} \cdots g_{n}$ with $g_{i} \in \widetilde{G}_{i}$ and $g_{i}$ unipotent (resp. semi-simple) in the algebraic sense (see e.g. [Sp] for a definition of these terms).

The Fitting subgroup of $G$ is the maximal, connected, normal, nilpotent, definable subgroup of $G$. A group of finite Morley rank $G$ has a unique Fitting

Received by the editors November 14, 1988 and, in revised form, February 21, 1989.

1980 Mathematics Subject Classification (1985 Revision). Primary 03C45, 03C60, $20 \mathrm{~F} 16$.

The second author's research was partially supported by NSF Grant DMS-8801021. The article was written at the University of Notre Dame while the second author was a visitor. He would like to thank the members of the mathematics department for their hospitality. 
subgroup that we denote by $F(G)$, or by $F$ if there is no possible confusion. With these notation and convention we prove the following result.

Theorem 2. Let $G, \widetilde{G}, \widetilde{G}_{i}$ be as in Theorem 1. Then

(i) $F(G)=F(\widetilde{G}) \cap G$.

(ii) $F(G)$ has a complement $V$ in $G: G=F \rtimes V$.

(iii) Elements of $F(G)$ are unipotent elements of $G$ (in $\widetilde{G}$ ).

(iv) If the characteristic of each base field $K_{i}$ of $\widetilde{G}_{i}$ is different from 0 , then $V$ is definable and its elements are semi-simple in $\widetilde{G}$.

We do not know if the elements of the complement $V$ are semi-simple (or if $V$ can be chosen semi-simple) in case the characteristic of one of the fields $K_{i}$ is zero.

Now a few words placing this article in the historical context: Gregory Cherlin [Ch] and Boris Zil'ber conjectured independently that infinite simple groups of finite Morley rank are algebraic groups over algebraically closed fields (known under the name of Cherlin's Conjecture). The conjecture is still open. Since maximal solvable connected subgroups (i.e., Borels) of a simple algebraic group are an important tool in their study and classification, one may legitimately think that solvable subgroups will also be important in the study of simple groups of finite Morley rank. This hypothetical hope is our main reason for studying solvable groups of finite Morley rank. A more down-to-earth reason is that centerless solvable groups of finite Morley rank seem to be easier to understand than the simple ones (thanks to Zil'ber's theorem [Zi] that states that in such a group one can interpret an algebraically closed field).

It will be interesting to generalize these theorems to arbitrary connected solvable but non-nilpotent groups (with or without center) of finite Morley rank. Another interesting problem is to classify simple $B-N$ pairs of rank 1 and of finite Morley rank with $B$ satisfying the hypothesis on $G$ of Theorem 1 . Should then $B$ be an algebraic group? (See e.g. [Car] for a definition of a $B-N$ pair). This problem is interesting for the following reason: what prevented us from proving that the group $G$ of Theorem 1 is a product of algebraic groups is the hypothetical existence of infinite proper definable multiplicative subgroups of an algebraically closed field of finite Morley rank; it would be interesting to know if this is still a problem in simple groups; in other words, is the existence of bad fields a serious obstacle to prove Cherlin's Conjecture? Thus the above problem of $B-N$ pairs of rank 1 would be a test-case.

The notation is as in [Ne]. The article is self-contained, however the reader who wants a better understanding of the involved machinery should be familiar (at least superficially) with $\S \S 5$ and 6 of that article. In any event all the results of [Ne] used in this article are stated (without proofs) in $\S \S 2$ and 3.

To prove Theorems 1 and 2 we need to understand the structure of $\widetilde{G}_{i}$ and its relevent properties explained in [Ne]. We give a survey of these results in $\S 2$. In $\S 3$ we explain the structure of local rings discovered by Cohen [Co]. In [E-N] 
we gave an easy proof of Cohen's structure theorem which makes the structure of such a ring more transparent. The last sections are devoted to the proofs of the theorems.

\section{The GROUPS $\widetilde{G}$ AND $\widetilde{G}_{i}$}

Let $G$ be a connected, centerless, solvable of class 2 group of finite Morley rank. Thus $G^{\prime}$ is abelian. By Theorem 1 of $[\mathrm{Ne}], G=G^{\prime} \rtimes T$ for some definable (so also connected and abelian) subgroup $T$. Since $G$ is centerless $T$ acts faithfully on the abelian group $G^{\prime}$. Therefore we may view $T$ as a subset of End $G^{\prime}$. Let $R$ be the ring generated by this action of $T$ in the endomorphism ring of $G^{\prime}$. If we write $G^{\prime}$ additively, it is easy to see that

$$
R=\mathbf{Z}[T] / \text { ann } G^{\prime}
$$

where elements of the group ring $Z[T]$ act on $G^{\prime}$ in a natural way: if $\sum n_{i} t_{i} \in$ $\mathbf{Z}[T]$ with $n_{i} \in \mathbf{Z}, t_{i} \in T$ and if $x \in G^{\prime}$ then

$$
\left(\sum n_{i} t_{i}\right)(x)=\sum n_{i} t_{i}(x)
$$

with the additive notation,

$$
\left(\sum n_{i} t_{i}\right)(x)=\prod\left(x^{n_{i}}\right)^{t_{i}}
$$

with the multiplicative notation. In this article we will use the additive notation. Notice that $G^{\prime}$ is an $R$-module. Let $\widetilde{G}$ be $G^{\prime} \rtimes R^{*}$ where $R^{*}$ denotes the multiplicative group of invertible elements of $R$. By [Ne, Proposition 26],

$$
R=\bigoplus_{1 \leq i \leq \ell} R_{i}
$$

for some specific infinite ideals $R_{i}$ of $R$. By [Ne, Lemma 29] each $R_{i}$ is a local ring with nilpotent maximal ideal $M_{i}$. By [Ne, Lemma 32], each $R_{i}$ is interpretable in $G$, thus they all have finite Morley rank. By Cherlin-Reineke [Ch-R], the maximal ideals $M_{i}$ are all definable and by Macintyre [Mac 2], $R_{i} / M_{i}$ is an algebraically closed field. Let $K_{i}=R_{i} / M_{i}$.

Let $G_{i}^{\prime}=R_{i} G^{\prime}$. Since $R_{i}$ is interpretable and $G^{\prime}$ definable, $G_{i}^{\prime}$ is a definable subgroup of $G$. Clearly $G^{\prime}=\bigoplus G_{i}^{\prime}$ and $R_{j}^{*}$ acts as identity on $G_{i}^{\prime}$ if $i \neq j$. So the group $\widetilde{G}$ defined as $G^{\prime} \rtimes R^{*}$ is canonically isomorphic to $\bigoplus_{1 \leq i \leq \ell}\left(G_{i}^{\prime} \rtimes R_{i}^{*}\right)$. We let $\widetilde{G}_{i}$ denote the group $G_{i}^{\prime} \rtimes R_{i}{ }^{*}$. Since $T<R^{*}$, we clearly have an interpretable imbedding of $G$ in $\widetilde{G}: G<\widetilde{G}$.

Let $S_{i 1}<S_{i 2}<\cdots<S_{i k}=G_{i}^{\prime}$ be the socle series of $G_{i}^{\prime}$. Thus by definition $S_{i 1}$ is the subgroup generated by the minimal, $G$-normal, definable subgroups of $\widetilde{G}_{i} . S_{i 1}$ is called the socle of $\widetilde{G}_{i}$. It is normal, connected and definable. $S_{i, j+1}$ is the unique subgroup containing $S_{i j}$ and having the following property: $S_{i, j+1} / S_{i j}$ is the socle of $\widetilde{G}_{i} / S_{i j} . S_{i j}$ is called the $j$ th socle of $\widetilde{G}_{i}$. Each quotient $S_{i, j+1} / S_{i j}$ is a direct sum of finitely many minimal definable 
minimal normal subgroups of $\widetilde{G}_{i} / S_{i j}$ and the action of $\widetilde{G}_{i} / S_{i j}$ on these minimal subgroups is definably isomorphic to the action of the multiplicative group $\left(R_{i} / M_{i}\right)^{*}$ on the additive group $\left(R_{i} / M_{i},+\right)$ by multiplication. These last sentences follow from [Ne, Corollary 9, Lemma 4] and the content of Zil'ber's Theorem explained at length in [Ne, §3]. By [Ne, Lemma 23], $M_{i} S_{i, j+1} \subseteq S_{i j}$. Since the elements of $R_{i}{ }^{*}$ are invertible, if $x \in S_{i, j+1} \backslash S_{i j}$ then $R_{i}{ }^{*} x \cap S_{i j}=\varnothing$.

We will come back to the structure of $\widetilde{G}_{i}$ later in $\S 3$, after we get more information about local rings $R_{i}$.

\section{THE RING $R_{i}$ AND MORE ABOUT $\widetilde{G}_{i}$}

Let us drop the subscript $i$ for the next two sections. Thus $\widetilde{G}, G, R, M$, $K, S_{j}, G^{\prime}, R^{*}$ denote $\widetilde{G}_{i}, G_{i}, R_{i}, M_{i}, K_{i}, S_{i j}, G_{i}^{\prime}, R_{i}^{*}$ respectively.

Each factor $M^{k} / M^{k+1}$ is a $K$-vector space; by finiteness of Morley rank they have finite dimension. Since $M$ is nilpotent, this shows that $M$ is a finitely generated ideal of $R$. Hence $M$ is definable. In fact by [Ne 1] every ideal of $R$ is definable, connected and finitely generated. In particular $R$ is Artinian and Noetherian.

We have to distinguish two cases: $\operatorname{char} R=0$ and $\operatorname{char} R=p^{m+1} \neq 0$.

If $\operatorname{char} R=0$ then since $M$ is nilpotent, char $K=0$ also. In this case (in fact if $\operatorname{char} K=\operatorname{char} R$ ) we can find a copy of $K$ in $R$ (not necessarily definable); then $R$ is isomorphic to $K\left[X_{1}, \ldots, X_{s}\right] / I$ where $I$ is an ideal containing a power of each monomial in $X_{1}, \ldots, X_{s} . M$ is also easy to recognize: $M \simeq\left\langle X_{1}, \ldots, X_{s}\right\rangle / I$. This is the easy case.

Suppose char $R=p^{m+1} \neq 0$. We will explain the structure of $R$ (see [Co] and also [E-N]). $1+M$ is a subgroup of $R^{*}$. It is also a pure subgroup of bounded exponent. Therefore it has a complement that we denote by $U$ :

$$
R^{*}=(1+M) \times U=U+M
$$

where $\times$ denotes the direct product sign in a multiplicative situation. It is easy to show that

$$
U \simeq R^{*} / 1+M \simeq K^{*} \text {. }
$$

Thus $U$ is a divisible group. Also it is 0 -definable (which is not the case if the characteristic is 0 ). In fact

$$
U=\left(R^{*}\right)^{p^{m}} \text {. }
$$

$U$ is closed under product but $U \cup\{0\}$ is not closed under addition (unless $m=0)$. Let us close it under addition, i.e. let us consider the subring $W$ of $R$ generated by $U$. Then

$$
W=U^{\prime}+p U^{\prime}+\cdots+p^{m} U^{\prime}
$$

where $U^{\prime}=U \cup\{0\}$. Furthermore the above "decomposition" has the following uniqueness property: if $u_{0}, \ldots, u_{m}$ and $v_{0}, \ldots, v_{m}$ are in $U^{\prime}$ and if $u_{0}+p u_{1}+\cdots+p^{m} u_{m}=v_{0}+\cdots+p^{m} v_{n}$ then $u_{j}=v_{j}$ for all $j=1, \ldots, m$. 
Notice also that $R=U^{\prime}+M$ and $R^{*}=U+M$ and we again have uniqueness. Since $U^{\prime}$ is in bijection with $K$, it follows that $W$ is in bijection with $K^{m+1}$.

$W$ is a complete local ring with maximal ideal $p W$. Also char $W=$ char $R=p^{m+1}$ and $W / p W \simeq K$. Given these data there is a unique such ring (see e.g. [Se, $\S 5$, Corollary of Proposition 10]). They are called Witt rings (not to be confused with the Witt rings of quadratic forms) and their structure is made explicit by Witt [W] and can also be found in [Jac 1] or [Jac 2]. However, we will not be concerned with their definition; for us it will be enough to know that if $W$ is viewed as the variety $K^{m+1}$ via the above decomposition then the addition and the multiplication of $W$ are given by polynomial maps (see e.g. [Jac 1, Theorem 8.25]). We will refer to this fact by saying that $W$ is an "algebraic ring".

Let us go back to $G^{\prime}$. We would like to have a better understanding of the action of $U$ on $G^{\prime}$ (if $\operatorname{char} R=0$, then take $U$ to be a copy of $K^{*}$ found in $R$ ). Let $S_{j+1} / S_{j}$ be one of the quotients considered in $\S 2$. We have already noticed that $S_{j+1} / S_{j}$ is a direct sum of finitely many $G / S_{j}$-minimal subgroups. Choose a non-zero representative from each one of those minimal subgroups, say $x_{1}, \ldots, x_{n}$. Thus

$$
S_{j+1} / S_{j}=\overline{R x}_{1} \oplus \cdots \oplus \overline{R x}_{n}
$$

where - means "modulo $S_{j}$ ". But $R=U^{\prime}+M$ and $M x_{j} \subseteq S_{j}$, thus

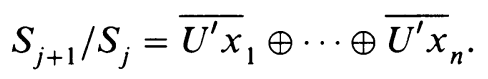

The reader should be aware that if char $R \neq 0, p$ then $U^{\prime} x_{k}$ is never a subgroup but only a unique set of representatives of $R x_{k}$ modulo $S_{j}$. Doing this for all $j$, we can find elements $x_{1}, \ldots, x_{n}$ of $G^{\prime}$ such that

$$
G^{\prime}=U^{\prime} x_{1}+\cdots+U^{\prime} x_{n}
$$

with the property that if $u_{1}, \ldots, u_{n} \in U^{\prime}, v_{1}, \ldots, v_{n} \in U^{\prime}$ and if $u_{1} x_{1}+$ $\cdots+u_{n} x_{n}=v_{1} x_{1}+\cdots+v_{n} x_{n}$, then $u_{j}=v_{j}$ for all $j=1, \ldots, n$. We will say that $x_{1}, \ldots, x_{n}$ is a $U^{\prime}$-base of $G^{\prime}$. Now it is clear how $U^{\prime}$ acts on $G^{\prime}$; it acts componentwise:

$$
u\left(u_{1} x_{1}+\cdots+u_{n} x_{n}\right)=u u_{1} x_{1}+\cdots+u u_{n} x_{n} .
$$

Since the product $u u_{j}$ depends polynomially on $u$ and $u_{j}$, this remark will be valuable to prove Theorem 1 . In fact at this point the proof Theorem 1 should be clear to the expert.

\section{4. $\widetilde{G}_{i}$ IS AN ALGEBRAIC GROUP}

We continue to omit the subscript $i$ as in the previous section.

By definition $\widetilde{G}=G^{\prime} \rtimes R^{*}$. Thus to prove Theorem 1 we need to show that $G^{\prime}$ and $R^{*}$ are algebraic groups and the action of $R^{*}$ on $G^{\prime}$ is an algebraic 
action. We fix a $U^{\prime}$-base $x_{1}, \ldots, x_{n}$ of $G^{\prime}$ constructed in the previous section. Let $p$ be the characteristic of $K . p$ may be 0 . For $x_{i}(i=1, \ldots, n)$ and $j$ an integer we can write

$$
p^{j} x_{i}=\sum_{k} \alpha_{i j k} x_{k}
$$

for some unique $\alpha_{i j k} \in U^{\prime}$. We fix $\alpha_{i j k}$ throughout this section. Notice that if $x_{i} \in S_{l}$, the $l$ th socle, then $p^{j} x_{i} \in S_{l-j}$ (because $M S_{l} \subseteq S_{l-1}$ and $p \in M$ ) and hence if $x_{i} \in S_{j}$ then $\alpha_{i j k}=0$ for all $k$.

Lemma 1. $G^{\prime}$ is an algebraic group.

Proof. We identify $G^{\prime}$ with the variety $K^{n}$ via the decomposition of $G^{\prime}$ with respect to the $U^{\prime}$-base $x_{1}, \ldots, x_{n}$ :

$$
G^{\prime}=U^{\prime} x_{1}+\cdots+U^{\prime} x_{n},
$$

remembering that $U^{\prime}$ is in (interpretable) bijection with $K$. Let

$$
x=\alpha_{1} x_{1}+\cdots+\alpha_{n} x_{n}, \quad y=\beta_{1} x_{1}+\cdots+\beta_{n} x_{n}
$$

be two elements of $G^{\prime}$. Then

$$
x+y=\tau_{1} x_{1}+\cdots+\tau_{n} x_{n}
$$

for some unique $\tau_{1}, \ldots, \tau_{n} \in U^{\prime}$. We need to show that each $\tau_{i}$ depends on $\alpha_{1}, \ldots, \alpha_{n}, \beta_{1}, \ldots, \beta_{n}$ polynomially, i.e. that there are $n$ polynomials $f_{1}, \ldots, f_{n}$, independent of $\alpha_{1}, \ldots, \alpha_{n}, \beta_{1}, \ldots, \beta_{n}$, with coefficients in $U^{\prime}(\simeq K)$ and in $2 n$ variables such that

$$
f_{i}\left(\alpha_{1}, \ldots, \alpha_{n}, \beta_{1}, \ldots, \beta_{n}\right)=\tau_{i}
$$

for each $i=1, \ldots, n$. We will prove this by induction on $S_{j}$ (the $j$ th socle of $G^{\prime}$ ).

Assume first that $x, y \in S_{1}$. We may delete $\alpha_{i} x_{i}$ 's, $\beta_{i} x_{i}$ 's for which $x_{i} \notin S_{1}$ to assume that each $x_{i}$ figuring in the above expression of $x$ or $y$ is in $S_{1}$. Then, with this convention, $p x_{i}=0$. Now compute

$$
x+y=\left(\sum_{i} \alpha_{i} x_{i}\right)+\left(\sum_{i} \beta_{i} x_{i}\right)=\sum_{i}\left(\alpha_{i}+\beta_{i}\right) x_{i} .
$$

Since $\alpha_{i}+\beta_{i}$ is not necessarily in $U^{\prime}$ we are not finished. But $\alpha_{i}+\beta_{i}$ is in $W$ and $W$ is an algebraic ring in the sense we gave to this term in the previous section. Thus

$$
\alpha_{i}+\beta_{i}=\sum_{j=0}^{m} \tau_{i j} p^{j}
$$

and each $\tau_{i j} \in U^{\prime}$ depends polynomially on $\alpha_{i}$ and $\beta_{i}$ (but the polynomial is independent of $\alpha_{i}$ and $\beta_{i}$ ). Putting this expression in the calculations of $x+y$ we obtain

$$
x+y=\sum_{i} \sum_{j} \tau_{i j} p^{j} x_{i}
$$


But with the convention of the beginning of this paragraph, $p^{j} x_{i}=0$ if $j \neq 0$. Thus

$$
x+y=\sum_{i} \tau_{i 0} x_{i}
$$

and we are done with the first step of the induction.

Let us assume we know how to calculate the sum of elements of $S_{i}$ in an algebraic way. Take $x$ and $y$ in $S_{i+1}$. Assume again, without loss of generality that if $x_{j} \notin S_{i+1}$ then $\alpha_{j} x_{j}, \beta_{j} x_{j}$ do not occur in the expressions of $x$ and $y$. Then separate the basis $x_{1}, \ldots, x_{n}$ into two parts, the ones that belong to $S_{i}$, the ones that do not:

$$
\begin{aligned}
& x=\sum \alpha_{k} x_{k}+\sum \alpha_{j} x_{j}, \\
& y=\sum \beta_{k} x_{k}+\sum \beta_{j} x_{j},
\end{aligned}
$$

where $x_{k} \in S_{i+1} \backslash S_{i}, x_{j} \in S_{i}$ with a lot of notational abuse. First add $\sum \alpha_{k} x_{k}$ and $\sum \beta_{k} x_{k}$ the way we did above, but this time $p x_{k} \in S_{i}$ for all $k$ :

$$
\sum \alpha_{k} x_{k}+\sum \beta_{k} x_{k}=\sum_{k} \tau_{k 0} x_{k}+\sum_{k} \sum_{l>0} \tau_{k l} p^{l} x_{k}
$$

Now $x+y$ is equal to the sum of $\sum_{k} \tau_{k 0} x_{k}$ and of three elements of $S_{i}$, namely of

$$
\sum \alpha_{j} x_{j}, \quad \sum \beta_{j} x_{j} \text { and } \quad \sum_{k} \sum_{l>0} \tau_{k l} p^{l} x_{k}=\sum_{m} \sum_{k} \sum_{l>0} \tau_{k l} \alpha_{k l m} x_{m} .
$$

By induction this second sum (of the above three elements) is algebraic; by the algebraicity of $W, \tau_{k 0}$ are polynomials. This proves the lemma.

Lemma 2. The action of $R^{*}$ on $G^{\prime}$ is an algebraic action.

Proof. $R^{*}=U+M$. We know that the action of $U$ on $G^{\prime}$ is componentwise (see the last paragraph of $\S 3$ ) and $G^{\prime}$ is an algebraic group. Thus it remains to prove that the action of $M$ on $G^{\prime}$ is algebraic. We will first choose a " $U^{\prime}$ base" of $M$, the same way we did for $G^{\prime}$. Remember that $M^{i} / M^{i+1}$ is a finite-dimensional vector space over $R / M$. Choose a set of representatives of $M^{i} / M^{i+1}$ for a $K$-base and do this for each $i$, to get a set of elements $y_{i}, \ldots, y_{r} \in M$ for which

$$
M=U^{\prime} y_{1}+\cdots+U^{\prime} y_{r}
$$

Then we have the usual "uniqueness" property: if $u_{1}, \ldots, u_{r}, v_{1}, \ldots, v_{r} \in U^{\prime}$ and if $u_{1} y_{1}+\cdots+u_{r} y_{r}=v_{1} y_{1}+\cdots+v_{r} y_{r}$ then $u_{i}=v_{i}$ for each $i$. With this decomposition and with what we already know about the action of $U^{\prime}$ on $G^{\prime}$ (see the end of $\S 3$ ) we reduce the problem to the algebraicity of the action of each $y_{i}$ on $G^{\prime}$. But this is clear. Let $x_{1}, \ldots, x_{n}$ be a $U^{\prime}$-basis of $G^{\prime}$ as in the proof of Lemma 1. Then

$$
y_{i} x_{j}=\sum_{k} \beta_{i j k} x_{k}
$$

for some fixed $\beta_{i j k} \in U^{\prime}(\simeq K)$. The lemma is now proved. 
Lemma 3. $R^{*}$ is an algebraic group.

Proof. Let $y_{1}, \ldots, y_{r}$ be as in Lemma 2. Thus

$$
R^{*}=U+U^{\prime} y_{1}+\cdots+U^{\prime} y_{r}
$$

in a "unique" way. Identify $R^{*}$ with the variety $K^{*} \times K^{r}$ via this decomposition. Let

$$
y_{i} y_{j}=\sum_{k} \gamma_{i j k} y_{k}
$$

for some unique $\gamma_{i j k}$. Now the proof of the lemma is as the previous lemmas and we omit it.

\section{Proof of Theorem 2}

Now we have to put back the subscripts. Notation is as in $\S \S 2$ and 3. Let

$$
\begin{aligned}
U & =\bigoplus_{i=1}^{n} U_{i}, \\
X & =\prod_{i=1}^{n}\left(1+M_{i}\right), \\
X_{b} & =\prod_{d}\left\{1+M_{i}: R_{i} \text { has characteristic } p \neq 0\right\}, \\
X_{d} & =\prod\left\{1+M_{i}: R_{i} \text { has characteristic } 0\right\} .
\end{aligned}
$$

Then $X=X_{b} \times X_{d}, X_{b}$ has bounded order, $X_{d}$ is divisible, $X_{b}$ and $X_{d}$ are definable and connected $\left(1+M_{i}^{k} / 1+M_{i}^{k+1} \simeq M_{i}^{k} / M_{i}^{k+1}\right.$ and the latter is a vector space over $\left.K_{i}\right)$. Notice that $X_{d}$ has no elements of finite order. It is clear that $G_{i}^{\prime} \rtimes\left(1+M_{i}\right)$ is the set of unipotent elements of $\widetilde{G}_{i}$ and it can be easily checked to be the Fitting subgroup of $\widetilde{G}_{i}$. Also $R^{*}=X \times U$. Thus

$$
\begin{aligned}
F(\widetilde{G}) & =G^{\prime} \rtimes X, \\
\widetilde{G} & =F(\widetilde{G}) \rtimes U .
\end{aligned}
$$

Since $U_{i} \simeq K_{i}^{*}, U$ is a divisible group. When we will speak about $\widetilde{G}$ as a group of finite Morley rank, we will mean it is so with the $G$-language. In other words we assume that $G$ is a definable subgroup of $\widetilde{G}$ even though it may not be in the pure language of groups.

By Macintyre [Mac 1], $T=B \oplus D$ where $T$ is a complement of $G^{\prime}, B$ has bounded order and $D$ is divisible. If $B^{k}=1$ then $D=T^{k}$ and so $D$ is definable and being divisible it is connected. But $B$ is not necessarily definable. We will replace $B$ with a definable subgroup. Let

$$
C=\left\{t \in T: t^{k}=1\right\} \text {. }
$$

Since $T$ is abelian, $C$ is a definable subgroup containing $B$. We will show that $T=C^{\circ} \oplus D$. 
Lemma 1. $C^{\circ} \subseteq F(G)$.

Proof. By [Ne, Theorem 6], $G / F$ is a divisible group of finite Morley rank. Since $C^{\circ} F / F$ has finite exponent, $C^{\circ} F / F$ is finite. But $C^{\circ} F$ is clearly connected, thus so is $C^{\circ} F / F$. Hence $C^{\circ} F=F, C^{\circ} \subseteq F$.

Lemma 2. $F(G) \subseteq F(\widetilde{G}) \cap G$.

Proof. $F(G)$ is a nilpotent, definable, connected subgroup of $G$. Since $\widetilde{G}$ is interpretable in $G$ and we consider $\widetilde{G}$ with the $G$-language, these properties are preserved in $\widetilde{G}$. It remains to show that $F(G) \triangleleft \widetilde{G}$. Since $G^{\prime} \subseteq F(G)$, $\widetilde{G}=G^{\prime} \rtimes R^{*}$ we have

$$
F(G)=G^{\prime} \rtimes\left(F(G) \cap R^{*}\right) .
$$

$G^{\prime}$ clearly normalizes $F(G)$. Let us show that $R^{*}$ also normalizes $F(G)=$ $G^{\prime} \rtimes\left(F(G) \cap R^{*}\right)$; this will show that $F(G) \triangleleft \widetilde{G} . R^{*}$ normalizes $G^{\prime}$ by its definition. Since $F(G) \cap R^{*} \subseteq R^{*}$ and $R^{*}$ is commutative, $R^{*}$ also normalizes $F(G) \cap R^{*}$. Thus $R^{*}$ normalizes $F(G)$.

Lemma 3. $C^{\circ} \subseteq X_{b}$.

Proof. We have $C^{\circ}<T<R^{*}=X \times U=X_{b} \times X_{d} U$. Let $\pi$ be the second projection map from $R^{*}$ onto $X_{d} U . \pi\left(C^{\circ}\right)$ is a subgroup of finite exponent of the divisible definable group $X_{d} U$. Thus $\pi\left(C^{\circ}\right)$ is finite. On the other hand $C^{\circ}$ being connected, so is its projection $\pi\left(C^{\circ}\right)$. Therefore $\pi\left(C^{\circ}\right)=1$ and $C^{\circ} \subseteq X_{b}$.

Lemma 4. $X_{b} \cap\left(X_{d} U D\right)=1$.

Proof. We have

$$
X_{d} U \subseteq X_{d} U D \subseteq R^{*}=X \times U=X_{b} \times X_{d} U,
$$

so

$$
X_{d} U D=\left(X_{b} \cap X_{d} U D\right) \times X_{d} U .
$$

Clearly $X_{b} \cap X_{d} U D$ is finite (one is bounded, the other one is divisible); thus, $X_{d} U$ is a subgroup of finite index of the divisible group $X_{d} U D$, so $X_{d} U=$ $X_{d} U D$ and hence $X_{b} \cap X_{d} U D=1$.

Lemma 5. $T=C^{\circ} \oplus D$.

Proof. By Lemmas 3 and $4, C^{\circ} \cap D=1$. Let us show that $C^{\circ} D=T$; then the lemma will be proved. Since $C / C^{\circ}$ is finite, so is $B / C^{\circ} \cap B$. Since $T=B D$ this shows that $\left[T:\left(B \cap C^{\circ}\right) D\right]$ is finite. But

$$
\left(B \cap C^{\circ}\right) D \subseteq C^{\circ} D \subseteq T,
$$

so also $\left[T: C^{\circ} D\right]$ is finite. $T$ being connected and $C^{\circ} D$ definable we get $T=C^{\circ} D$. 


\section{Lemma 6.}

(i) $F(G)=F(\widetilde{G}) \cap G$.

(ii) $F(G)=G^{\prime} C^{\circ} \rtimes(D \cap F(\widetilde{G}))$.

(iii) $D \cap F(\widetilde{G})$ is a divisible group.

(iv) $D \cap F(\widetilde{G}) \subseteq X_{d}$.

Proof. Clauses (ii), (iii) and (iv) will be proved during the proof of (i). By Lemma 2 , to prove (i), we only need to show that $F(\widetilde{G}) \cap G \subseteq F(G)$. Now, we have

$$
G^{\prime} \subseteq F(\widetilde{G}) \cap G \subseteq G=G^{\prime} \rtimes T
$$

therefore

$$
F(\widetilde{G}) \cap G=G^{\prime} \rtimes(T \cap F(\widetilde{G}) \cap G)=G^{\prime} \rtimes(T \cap F(\widetilde{G})) .
$$

Thus we just need to prove that $T \cap F(\widetilde{G}) \subseteq F(G)$. But $T=C^{\circ} \oplus D$ and $C^{\circ} \subseteq F(G) \subseteq F(\widetilde{G})$; thus,

$$
T \cap F(\widetilde{G})=C^{\circ} \oplus(D \cap F(\widetilde{G})) .
$$

Since $C^{\circ} \subseteq F(G)$, we just need to prove that $D \cap F(\widetilde{G}) \subseteq F(G)$. Also $D<$ $T<R^{*}, R^{*} \cap G^{\prime}=1$ and $X \subseteq R^{*}$, so

$$
D \cap F(\widetilde{G}) \subseteq R^{*} \cap\left(G^{\prime} \rtimes X\right)=R^{*} \cap X=X .
$$

Now it follows from Lemma 4 that $D \cap F(\widetilde{G}) \subseteq X_{d}$ (which is iv)). We distinguish two cases:

Case 1. $D \cap F(\widetilde{G})$ is infinite. Then, being an infinite subgroup of the divisible group $D, D \cap F(\widetilde{G})$ is divisible and hence connected. (2) shows that then $T \cap F(\widetilde{G})$ is connected and by $(1), F(\widetilde{G}) \cap G$ is connected. Since $F(\widetilde{G}) \cap G$ is clearly definable, nilpotent and normal, with its connectedness we obtain $F(\widetilde{G}) \cap G \subseteq F(G)$, proving the Lemma in Case 1 .

Case 2. $D \cap F(\widetilde{G})$ is finite. By (3) $D \cap F(\widetilde{G}) \subseteq X=X_{b} X_{d}$. Since $D \cap F(\widetilde{G})$ has finite exponent and $X_{d}$ is torsion-free we have $D \cap F(\widetilde{G}) \subseteq X_{b}$. Thus

$$
D \cap F(\widetilde{G}) \subseteq X_{b} \cap D,
$$

but $X_{b} \cap D=1$ by Lemma 4 , so $D \cap F(\widetilde{G})=1$. By (2) $T \cap F(\widetilde{G})=C^{\circ} \subseteq F(G)$ and the Lemma is now proved.

Lemma 7. Elements of $F(G)$ are the unipotent elements of $G$.

Proof. Since this is true for $F(\widetilde{G})$, the lemma follows from the previous one.

Lemma 8. $G=F(G) \rtimes V$ for some divisible abelian group $V$ of finite Morley rank.

Proof. Since $G=G^{\prime} \rtimes T=G^{\prime} C^{\circ} \rtimes D$, by Lemma 6 (ii) we need to prove that $X_{d} \cap D$ splits in $D$. But by Lemma 6 (iii) and (iv) $X_{d} \cap D$ is divisible. Thus $D=\left(X_{d} \cap D\right) \oplus V$ for some subgroup $V$ (see, e.g., [Kap]). Now we have 
$G=F(G) \rtimes V$. Since $G / F(G)$ is divisible and $V \simeq G / F(G)$ the rest of the Lemma is trivial.

It remains to prove (iv) of Theorem 2. If char $K_{i} \neq 0$ for all $i$, then $X_{d}=1$ and thus, from Lemma 6 (iv) and (ii) we get

$$
F(G)=G^{\prime} C^{\circ} \text {. }
$$

Since we know that $G=\left(G^{\prime} C^{\circ}\right) \rtimes D$, we may take $V$ to be $D$ which is definable. Let us show that elements of $D$ are semi-simple, i.e., that $D \subseteq U$. We have

$$
U \subseteq D U \subseteq R^{*}=X \times U
$$

thus $D U=(D U \cap X) \times U$. But $X$ has finite exponent this time. Thus $D U \cap X$ is finite (because $D U$ is divisible). Therefore $U$ is a subgroup of finite index of the divisible group $D U$, so $U=D U, D \subset U$. This proves Theorem 2 completely.

\section{REFERENCES}

[Car] R. W. Carter, Simple groups of Lie type, 1972.

[Ch] G. Cherlin, Groups of small Morley rank, Ann. of Math. Logic 17 (1979), 1-28.

[Ch-R] G. Cherlin and J. Reineke, Stability and categoricity for commutative rings, Ann. of Math Logic 10 (1976), 376-399.

[Co] I. S. Cohan, On the structure and ideal theory of complete local rings, Trans. Amer. Math. Soc. 59 (1936), 54-106.

[E-N] K. Enochs and A. Nesin, A note on complete local rings, preprint.

[Jac 1] N. Jacobson, Basic algebra II, Freeman and Company, 1980.

[Jac 2] _ Structure of rings, Amer. Math. Soc. Colloquium Publications, vol. XXXVII, 1964.

[Kap] I. Kaplansky, Infinite abelian groups, Univ. of Michigan Press, 1954.

[Mac 1] A. Macintyre, On $\omega_{1}$-categorical theories of abelian groups, Fund. Math. 70 (1971), 253270.

[Mac 2] _ On $\omega_{1}$-categorical theories of fields, Fund. Math. 71 (1971), 1-25.

[Ne] A. Nesin, On solvable groups of finite Morley rank, Trans. Amer. Math. Soc. (to appear).

[Ne 1] _ Non-associative rings of finite Morley rank, Model Theory of Groups, Notre Dame Mathematical Lecture Series (A. Nesin and A. Pillay, eds.), 1979.

[Se] J.-P. Serre, Corps locaux, Publications de l'Institut de Mathématique de l'Université de Nancago, VIII, Hermann, Paris, 1968.

[Sp] T. A. Springer, Linear algebraic groups, Birkhäuser, 1981.

[W] E. Witt, Zyklische Körper und Algebren der Charakteristik p, J. Reine Angew. Math. 176, 126-140.

[Zi] B. Zil'ber, Some model theory of simple algebraic groups over algebraically closed fields, Colloq. Math. (2) 48 (1984).

Department of Mathematics, University of Notre Dame, Notre Dame, Indiana 46556

Department of Mathematics, University of California, Irvine, California 92717 